\title{
A Magneto-convection Over a Semi -infinite Porous Plate with Heat Generation
}

\author{
T. Raja ${ }^{1 \dagger}$, S.Karthikeyan ${ }^{2}$ and B. Senthilnathan ${ }^{3}$ \\ ${ }^{1}$ Department of Mathematics, Mahendra Engg. College, Namakkal, 637503, India \\ ${ }^{2}$ Department of Mathematics, Erode Arts and Science College, Erode, 638009, India \\ ${ }^{3}$ Department of Mathematics, Jansons Institute of Technology, Coimbatore, 641659, India \\ †Corresponding Author Email: rajaski88@gmail.com
}

(Received July 31, 2012; accepted October 17, 2012)

\begin{abstract}
Convective flow through porous media is a branch of research undergoing rapid growth in fluid mechanics and heat transfer. This is quite natural because of its important applications in environmental, geophysical and energy related engineering problems. Prominent applications are the utilization of geothermal energy, the control of pollutant spread in ground water, the design of nuclear reactors, solar power collectors and the heat transfer associated with the deep storage of nuclear waste. The study of heat generation in moving fluids is important in problems dealing with chemical reactions and those concerned with dissociating fluids. Heat generation effects may alter the temperature distribution and this in turn can affect the particle deposition rate in nuclear reactors, electronic chips and semi conductor wafers. Although exact modeling of internal heat generation is quite difficult, some simple mathematical models can be used to express its general behaviour for most physical situations. The objective of this work is to investigate the effects of internal heat generation on an unsteady two-dimensional magnetohydrodynamic free convection flow of a viscous, incompressible fluid free convection flow past a semi-infinite vertical porous plate embedded in a porous medium, in the presence of variable suction. The equations of continuity, linear momentum and energy, which govern the flow field, are transformed to a system of ordinary differential equations by perturbation technique. The resulting equations are solved analytically to obtain the solutions for the velocity and temperature fields. The behavior of the velocity, temperature, skin-friction and Nusselt number have been discussed for variations in the physical parameters.
\end{abstract}

Keywords: Magneto-convection, Porous plate.

NOMENCLATURE

$\begin{array}{ll}A & \text { suction velocity parameter } \\ B_{0} & \text { magnetic induction } \\ C_{f} & \text { skin-friction coefficient } \\ G_{c} & \text { solutal Grashof number } \\ G & \text { thermal Grashof number } \\ \mathrm{g} & \text { acceleration due to gravity } \\ \mathrm{K} & \text { permeability of the porous medium } \\ \mathrm{k} & \text { thermal conductivity } \\ \mathrm{M} & \text { magnetic field parameter } \\ \mathrm{N} & \text { dimensionless material parameter } \\ P_{r} & \text { Prandtl number } \\ Q_{0} & \text { heat absorption coefficient } \\ \mathrm{Re}_{x} & \text { local Reynolds number } \\ \mathrm{T} & \text { temperature }\end{array}$

$\begin{array}{ll}\mathrm{t} & \begin{array}{l}\text { dimensionless time } \\ U_{0}\end{array} \\ \mathrm{u}, \mathrm{v} & \begin{array}{l}\text { scale of free stream velocity } \\ \text { components of velocities along and } \\ \text { perpendicular to the plate respectively }\end{array} \\ V_{0} & \begin{array}{l}\text { scale of suction velocity. } \\ \alpha\end{array} \\ \beta & \text { fluid thermal diffusivity } \\ \eta & \text { coefficient of volumetric thermal expansion } \\ \phi & \text { dimensionless normal distance } \\ \sigma & \text { fluid electrical conductivity } \\ \rho & \text { fluid density } \\ \mu & \text { fluid dynamic viscosity } \\ \nu & \text { fluid kinematic viscosity } \\ \tau & \text { friction coefficient } \\ \theta & \text { dimensionless temperature }\end{array}$




\section{INTRODUCTION}

Natural convection in porous media has gained significant attention in recent years due to its diversified applications in geophysics and energy related engineering problems such as in isothermal reservoirs, heat storage beds, aquifers, porous insulation, extraction of geothermal energy and grain storage. Magnetohydrodynamics (MHD) plays a vital role in agriculture, engineering and petroleum industries. The study of MHD natural convection flow and heat transfer of an electrically conducting fluid in a heated semi-infinite vertical porous movable plate finds useful applications in many engineering problems such as MHD generator, plasma studies, nuclear reactors and the boundary layer control in the field of aeronautics and aerodynamics. Muthucumaraswamy and Ganesan (1998) have presented a finite-difference solution for an unsteady flow in an impulsively started vertical plate with heat and mass transfer. They reported that there is a rise in the velocity due to the presence of a mass diffusion and the velocities decreases with in increases of Schmidt number leads to a fall in the velocity. Kim (2000) studied the unsteady two-dimensional laminar flow of a viscous incompressible electrically conducting fluid in the vicinity of a semi-infinite vertical porous moving plate in the presence of a transverse magnetic field. The effect of radiation on MHD steady asymmetric flow of an electrically conducting fluid is a stretching porous sheet in the presence of radiation is studied by Ouaf (2005). Exact solutions for the velocity and temperature fields' effect have been derived. An investigation has done to study the effects of variable viscosity and thermal conductivity on the unsteady two-dimensional laminar flow of a viscous incompressible conducting fluid in a semi-infinite porous moving plate taking into account the effect of a magnetic field in the presence of variable suction by Seddeek and Salama (2007).

It reframe indicates that the velocity increases with the increase in variable viscosity, thermal conductivity, the exponential index, Grashof number but it decreases as the magnetic field parameter increases. Earlier reports shows that do not deal with internal heat generation. Chamkha et al. $(1998,1999)$ have been extensively studied about unsteady flow and heat transfer on a semi-infinite flat plate. Most of the previous studies have not dealt with the internal heat generation. In the present work an attempt has been made to extend the earlier works of Kim (2000), Seddeek and Salama (2007) and Suneetha (2011) by investigating the effects of internal heat generation to the unsteady MHD flow in a semi-infinite vertical moving plate with variable suction.

\section{Mathematical Analysis}

Consider unsteady two-dimensional flow of a laminar, incompressible, viscous, electrically conducting and heat-generating fluid past a semi-infinite vertical permeable moving plate embedded in a uniform porous medium and subjected to a uniform transverse magnetic field in the presence of thermal buoyancy effects. $\frac{\partial v^{*}}{\partial y^{*}}=0$

Momentum:

$$
\begin{aligned}
& \frac{\partial u^{*}}{\partial t^{*}}+v^{*} \frac{\partial u^{*}}{\partial y^{*}}=-\frac{1}{\rho} \frac{\partial p^{*}}{\partial x^{*}}+v \frac{\partial^{2} u^{*}}{\partial y^{* 2}}+ \\
& g \beta\left(T-T_{\infty}\right)-v \frac{u^{*}}{K^{*}}-\frac{\sigma}{\rho} B_{0}{ }^{2} u^{*}
\end{aligned}
$$

Energy:

$\frac{\partial T}{\partial t^{*}}+v * \frac{\partial^{2} T}{\partial y^{* 2}}=\alpha \frac{\partial^{2} T}{\partial y^{* 2}}+\frac{Q_{0}}{\rho C_{p}}\left(T-T_{\infty}\right)$

where $\mathrm{x}^{*}, \mathrm{y}^{*}$ are dimensional distances along and perpendicular to the plate, $t^{*}$, dimensional time $u^{*}, v^{*}$, components of the dimensional velocities along $x^{*}$ and $y^{*}$ directions respectively. $\rho$ is fluid density, $v$, kinematic viscosity, $\sigma$, the fluid electrical conductivity, $B_{0}, \quad$ Magnetic induction, $K^{*}$, permeability of the porous medium, $T$ dimensional temperature, $Q_{0}$, dimensional heat generation coefficient, $\alpha$, the fluid thermal diffusivity, $g$, gravitational acceleration and $\beta$, thermal expansion coefficient.

The boundary conditions for the velocity and temperature fields are

$u^{*}=u^{*}$

$T=T_{\omega}+\varepsilon\left(T_{\omega}-T_{\infty}\right) e^{n^{*} t^{*}} \quad$ at $y^{*}=0$

$u^{*} \rightarrow U_{\infty}^{*}=U_{0}\left(1+\varepsilon e^{n^{*} t^{*}}\right)$

$T \rightarrow T_{\infty} \quad$ as $y^{*} \rightarrow \infty$

where $u_{p}^{*}$ is wall dimensional velocity, $T_{\omega}$, wall dimensional temperature, $U_{\infty}^{*}$, free stream dimensional velocity, $T_{\infty}$, free stream dimensional temperature, $U_{0}$ and $n^{*}$ are constants.

It is clear from Eq. (1) that the suction velocity at the plate surface is a function of time only. Assuming that it takes the following exponential form

$v^{*}=-V_{0}\left(1+\varepsilon A e^{n^{*} t^{*}}\right)$

where $A$ is real positive constant, $\varepsilon$ and $\varepsilon A$ are small less than unity, $V_{0}$ is scale of suction velocity which has non-zero positive constant. Outside the boundary layer of equation:

Continuity: 
T. Raja et al. / JAFM, Vol. 6, No. 4, pp. 589-595, 2013.

$-\frac{1}{\rho} \frac{d p^{*}}{d x^{*}}=\frac{d U_{\infty}^{*}}{d t^{*}}+\frac{v}{K^{*}} U_{\infty}^{*}+\frac{\sigma}{\rho} B_{0}^{2} U_{\infty}^{*}$

It is convenient to employ the following dimensionless variables.

$u=\frac{u^{*}}{U_{0}}, \quad \mathrm{v}=\frac{v^{*}}{V_{0}}, \quad \mathrm{y}=\frac{V_{0} y^{*}}{v}$,

$U_{\infty}=\frac{U_{\infty}^{*}}{U_{0}}, \quad U_{p}=\frac{u_{p}^{*}}{U_{0}}, \quad t=\frac{t V_{0}^{2}}{v}$,

$\theta=\frac{T-T_{\infty}}{T_{\omega}-T_{\infty}}, \quad \eta=\frac{\eta^{*} v}{V_{0}^{2}}, \quad \mathrm{~K}=\frac{K V_{0}^{2}}{v^{2}}$,

$M=\frac{\sigma B_{0}^{2} v}{\rho V_{0}^{2}}, \quad \varphi=\frac{v Q_{0}}{\rho C_{p} V_{0}^{2}}$,

$G=\frac{v \beta g\left(T_{\omega}-T_{\infty}\right)}{U_{0} V_{0}^{2}}$

Substituting Eq. (7) and Eq. (8) in Eq. (2), we get

$\frac{\partial u}{\partial t}-\left(1+\varepsilon A e^{n t}\right) \frac{\partial u}{\partial \eta}$

$=\frac{d U_{\infty}}{d t}+\frac{\partial^{2} u}{\partial \eta^{2}}+G \theta+N\left(U_{\infty}-u\right)$

where $\mathrm{N}=\left(\mathrm{M}+\frac{1}{\mathrm{~K}}\right)$.

Again substituting Eq. (7) and Eq. (8) in Eq. (3) we get

$$
\frac{\partial \theta}{\partial t}-\left(1+\varepsilon A e^{n t}\right) \frac{\partial \theta}{\partial \eta}=\frac{1}{P_{r}} \frac{\partial^{2} \theta}{\partial \eta^{2}}+\varphi \theta
$$

where $G$ is thermal grashof number, $P_{r}$, Prandtl number and $\varphi$, dimensionless heat generation coefficient.

The dimensionless form of the boundary conditions Eq. (4) and Eq. (5) become:

$u=U_{p}, \quad \theta=1+\varepsilon e^{n t}$ at $\quad \eta=0$

$u \rightarrow U_{\infty}, \quad \theta \rightarrow 0$ as $\eta \rightarrow \infty$

\subsection{Solution of the Problem}

Equations (10), (11) and (12) represent a set of partial differential equations that cannot be solved in closed form. However, it can be reduced to a set of ordinary differential equations in dimensionless form that can be solved analytically. This can be done by representing the velocity and temperature as:

$u=f_{0}(\eta)+\varepsilon e^{n t} f_{1}(\eta)+o\left(\varepsilon^{2}\right)+\ldots \ldots \ldots$

and

$\theta=g_{0}(\eta)+\varepsilon e^{n t} g_{1}(\eta)+o\left(\varepsilon^{2}\right)+\ldots \ldots \ldots$.

Substituting Eq. (13) in Eq. (9), we get

$$
\begin{aligned}
& \varepsilon e^{n t}\left[n f_{1}(\eta)-f_{1}^{\prime}(\eta)-A f_{0}^{\prime}(\eta)\right. \\
& \left.-f_{1}^{\prime \prime}(\eta)-G g_{1}(\eta)-n-N+N f_{1}\right] \\
& -\left[f_{0}^{\prime}(\eta)+f_{0}^{\prime \prime}(\eta) G g_{0}+N-N f_{0}\right]=0
\end{aligned}
$$

Equating the harmonic and non-harmonic terms and neglecting the higher order terms of $0\left(\varepsilon^{2}\right)$ in Eq. (15), we get

$f_{1}^{\prime \prime}+f_{1}^{\prime}-(N+n) f_{1}=-A f_{0}^{\prime}-G g_{1}-(N+n)$

and

$f_{0}^{\prime \prime}+f_{0}^{\prime}-N f_{0}=-N-G g_{0}$

Substituting Eq. (14) in Eq. (10), we get

$$
\begin{aligned}
& n \varepsilon e^{n t} g_{1}(\eta)-\varepsilon e^{n t} g_{1}{ }^{\prime}(\eta)+\varepsilon e^{n t} A g o \\
&+\frac{1}{P_{r}} \varepsilon e^{n t} g_{1}{ }^{\prime \prime}(\eta)+\varphi \varepsilon e^{n t} g_{1}(\eta) \\
&= g_{0}{ }^{\prime}(\eta)+\frac{1}{P_{r}} g_{0}^{\prime \prime}(\eta)+\varphi g_{0}(\eta) \\
& \varepsilon e^{n t}\left[n g_{1}(\eta)-g_{1}^{\prime}(\eta)-A g_{0}{ }^{\prime}(\eta)-\frac{1}{P_{r}} g_{1}^{\prime \prime}(\eta)\right. \\
&\left.+\varphi g_{1}(\eta)\right] \\
&=g_{0}^{\prime}(\eta)+\frac{1}{P_{r}} g_{0}^{\prime \prime}(\eta)+\varphi g_{0}(\eta)
\end{aligned}
$$

Equating the harmonic and non-harmonic terms and neglecting $O\left(\varepsilon^{2}\right)$ in Eq. (19) we get

$$
\begin{aligned}
& n g_{1}-g_{1}^{\prime}-A g_{0}^{\prime}-\frac{1}{P_{r}} g_{1}^{\prime \prime}+\varphi g_{1}=0 \\
& g_{1}^{\prime \prime}+P_{r} g_{1}^{\prime}-n P_{r} g_{1}-P_{r} \varphi g_{1}=-A P_{r} g_{0}^{\prime}
\end{aligned}
$$

and

$$
\begin{aligned}
& g_{0}^{\prime}+\frac{1}{P_{r}} g_{0}^{\prime \prime}+\varphi g_{0}=0 \\
& g_{0}^{\prime \prime}+P_{r} g_{0}^{\prime}+P_{r} \varphi g_{0}=0
\end{aligned}
$$

First we solve Eq. (21) analytically, $g_{0}^{\prime \prime}+P_{r} g_{0}^{\prime}+P_{r} \varphi g_{0}=0$. The solution is $g_{0}(\eta)=A e^{-\frac{1}{2}\left[p_{r}+\sqrt{p_{r}{ }^{2}-4 p_{r} \phi}\right]} \eta e^{-\frac{1}{2}\left[-p_{r}+\sqrt{\left.p_{r}{ }^{2-4 p_{r} \phi}\right]} \eta\right.}$

The Boundary conditions are $g_{0}=1$ at $\eta=o$ and $g_{0} \rightarrow 0$ as $\eta \rightarrow \infty$.

Substituting the Boundary conditions in the above equation we get

$g_{0}=e^{-m_{1} \eta}$

Next we solve Eq. (20)

$g_{1}^{\prime \prime}+P_{r} g_{1}^{\prime}-n P_{r} g_{1}-P_{r} \varphi g_{1}=-A P_{r} g_{0}^{\prime}$. 
T. Raja et al. / JAFM, Vol. 6, No. 4, pp. 589-595, 2013.

Substituting Eq. (22) in Eq. (20), we get $g_{1}^{\prime \prime}+P_{r} g_{1}^{\prime}-(n+\varphi) P_{r} g_{1}=m_{1} A P_{r} e^{-m_{1} \eta}$.

The auxiliary equation is $m^{2}-P_{r} m-(n+\varphi)=0$.

$$
\begin{aligned}
C . F= & B e^{-m_{3} \eta}+C e^{\frac{1}{2}\left[-P_{r}+\sqrt{P_{r}^{2}+4(n+\varphi)}\right]} \eta \\
P . I= & A_{2} e^{-m_{1} \eta} \\
g_{1}(\eta) & =C . F+P I \\
& =B e^{-m_{3} \eta}+C e^{\frac{1}{2}\left[-P_{r}+\sqrt{P_{r^{2}}+4(\varphi+n)}\right] \eta} \\
& +A_{2} e^{-m_{1} \eta}
\end{aligned}
$$

The boundary conditions are $g_{1}=1$ at $\eta=0$ and $g_{0} \rightarrow 0$ as $\eta \rightarrow \infty$.

Substituting the boundary conditions in the above equation, we get

$$
g_{1}(\eta)=\left(1-A_{2}\right) e^{-m_{3} \eta}+A_{2} e^{-m_{1} \eta}
$$

Next we consider Eq. (17), $f_{0}^{\prime \prime}+f_{0}^{\prime}-N f_{0}=-N-G g_{0}$

The auxiliary equation is $m^{2}+m-N=o$.

$$
\begin{aligned}
C . F & =B e^{-\lambda_{1} \eta}+C e^{\frac{1}{2}[-1+\sqrt{1+4 N]} \eta} \\
P I_{1} & =1 \\
P I_{2} & =B_{1} e^{-m_{1} \eta} \\
f_{0}(\eta) & =B e^{-\lambda_{1} \eta}+C e^{\frac{1}{2^{2}[-1+\sqrt{1+4 N]} \eta}+B_{1} e^{-m_{1} \eta}}
\end{aligned}
$$

The boundary conditions are $f_{0}=U_{p}$ at $\eta=o$ and $f_{0}=1 \quad$ as $\eta \rightarrow \infty$.

Substituting the boundary conditions in the above equation we get

$$
f_{0}=1+C_{3} e^{-\lambda_{1} \eta}+B_{1} e^{-m_{1} \eta}
$$

Finally, we solve Eq. (17)

$$
f_{1}^{\prime \prime}+f_{1}^{\prime}-(N+n) f_{1}=-A f_{0}^{\prime}-G g_{1}-(N+n)
$$

The auxiliary equation

$$
\begin{aligned}
& m^{2}+m-(N+n)=o \\
& C . F=B e^{-\lambda_{3} \eta}+C e^{\frac{1}{2}[-1+\sqrt{1+4(N+n)} \eta} \\
& P I_{1}=G_{3} e^{-\lambda_{1} \eta} \\
& P I_{3}=B_{3} e^{-m_{3} \eta} \\
& P I_{2}+P I_{4}=D_{3} e^{-m_{1} \eta} \\
& P I_{5}=1
\end{aligned}
$$

$$
\begin{aligned}
f_{1}(\eta) & =C F+P I_{1}+P I_{2}+P I_{3}+P I_{4}+P I_{5} \\
& =B e^{-\lambda_{3} \eta}+C e^{\frac{1}{2}[-1+\sqrt{1+4(N+n)}]} \eta \\
& +G_{3} e^{-\lambda_{1} \eta}+B_{3} e^{-m_{1} \eta}+D_{3} e^{-m_{1} \eta}+1
\end{aligned}
$$

The boundary conditions are

$f_{1}=o$ at $\eta=o$

$f_{1}=1$ as $\eta \rightarrow \infty$

Substituting the boundary condition of the above equation, we get

$$
\begin{aligned}
f_{1}=C_{1} & +B_{3} e^{-m_{3} \eta}+D_{3} e^{-m_{1} \eta} \\
& +C_{4} e^{-\lambda_{3} \eta}+G_{3} e^{-\lambda_{1} \eta}
\end{aligned}
$$

where

$$
\begin{aligned}
& m_{1}=\frac{P_{r}+\sqrt{P_{r}^{2}-4 P_{r} \varphi}}{2} \\
& m_{3}=\frac{P_{r}+\sqrt{P_{r}^{2}-4(\varphi+n)}}{2} \\
& \lambda_{1}=\frac{1+\sqrt{1+4 N}}{2} \\
& \lambda_{3}=\frac{1+\sqrt{1+4(N}+n)}{2} \\
& C_{3}=U_{p}-1-B_{1} \\
& B_{1}=\frac{G}{-m_{1}^{2}+m_{1}+N} \\
& A_{2}=\frac{m_{1}}{m_{1}^{2}-P_{r} m_{1}-(\varphi+n)} \\
& C_{4}=-\left(1+B_{3}+D_{3}+G_{3}\right) \\
& B_{3}=\frac{-G\left(1-A_{2}\right)}{m_{3}^{2}-m_{3}-(N+n)} \\
& D_{3}=\frac{-G A_{2}+A m_{1} B_{1}}{m_{1}^{2}-m_{1}-(N+n)} \\
& G_{3}=\frac{A \lambda_{1} C_{3}}{\lambda_{1}^{2}-\lambda_{1}-(N+n)}
\end{aligned}
$$

Hence, we obtain the solution for the velocity equation as

$$
\begin{aligned}
u(\eta, t)= & f_{0}(\eta)+\varepsilon e^{n t} f_{1}(\eta) \\
u(\eta, t) & =1+C_{3} e^{-\lambda_{1} \eta}+B_{1} e^{-m_{1} \eta} \\
& +\varepsilon e^{n t}\left(1+C_{4} e^{-\lambda_{3} \eta}+B_{3} e^{-m_{3} \eta}\right. \\
& \left.+D_{3} e^{-m_{1} \eta}+G_{3}^{-\lambda_{1} \eta}\right)
\end{aligned}
$$

and the solution for the temperature as

$$
\begin{aligned}
& \theta(\eta, t)=g_{0}(\eta)+\varepsilon e^{n t} g_{1}(\eta) \\
& \theta(\eta, t)=e^{-m_{1} \eta}+\varepsilon e^{n t}\left[\left(1-A_{2}\right) e^{-m_{3} \eta}+A_{2} e^{-m_{1} \eta}\right]
\end{aligned}
$$




\section{RESUlTS AND DiscuSSIONS}

We have formulated and solved the problem of the effect of variable suction and heat generation on a MHD convective heat transfer past a semi-infinite moving plate. Based on these solutions, we have carried out numerical computations for the velocity and temperature for values of the material parameters. The boundary condition $y \rightarrow \infty$ is approximated by $y_{\max }=6$, which is sufficiently large for the velocity to approach the relevant stream velocity.

Figure 1 illustrates the temperature profiles with spanwise co-ordinate y for different Prandtl numbers. Here we take $n=0.1, t=1, \varepsilon=0.2, U_{p}=0.5$, $A=0.5, \quad S=-1.0, \quad G r=2.0, \quad K=0.5 \quad$ and $M=2.0$.

We have plotted the curves for temperature for different values of Prandtl number $\operatorname{Pr}$ such as $\operatorname{Pr}=0.7,0.1$ and 6.7. $\operatorname{Pr}=0.7$ corresponds to gases, $\operatorname{Pr}=1.0$ corresponds to air and $\operatorname{Pr}=6.7$ corresponds to water. Figure 1 depicts that as the values of Pr increases from 0.7 to 6.7, we can find that there is a decrease in the temperature profile and hence there is a decrease in thermal boundary layer thickness and more uniform temperature distribution across the boundary layer.

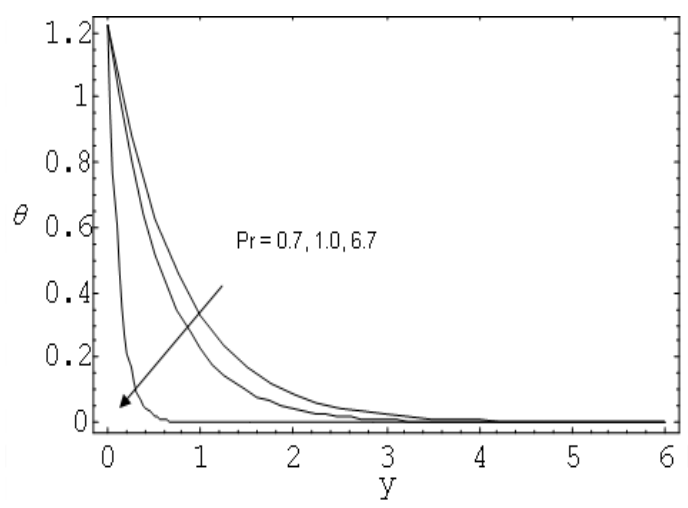

Fig. 1. Temperature profiles for different Prandtl numbers with $n=0.1, t=1, \varepsilon=0.2, U_{p}=0.5$,

$$
\begin{gathered}
A=0.5, S=-1.0, G r=2.0 K=0.5 \text { and } \\
M=2.0
\end{gathered}
$$

Figure 2 shows the temperature profiles for different heat generation parameters with $n=0.1, t=1$, $\varepsilon=0.2, U_{p}=0.5, A=0.5, \operatorname{Pr}=0.7, G r=2.0$, $K=0.5$ and $M=2.0$.

From this figure, we conclude that the temperature decreases gradually as the heat generation parameter $\mathrm{S}$ increases from zero. When $\mathrm{S}$ takes 2 , we see that the temperature is lowest and there is a uniform temperature distribution across the boundary layer. This result agrees with the natural phenomenon. From Fig. 3. we observe that the increasing Prandtl numbers results in decreasing velocity. When Pr takes 0.7 (which the Prandtl number is for gases), velocity starts from zero near to the boundary layer, increases rapidly and attains its peak. Then it gradually decreases before attaining the free stream velocity. The velocity profiles for different Grashof numbers are depicted in Fig. 4 we plot the temperature profile for $\mathrm{Gr}=1,5$ and 10 . We observe from this figure that when Grashof number increases, velocity attains in peak value and decreases suddenly before reaching the free stream velocity.

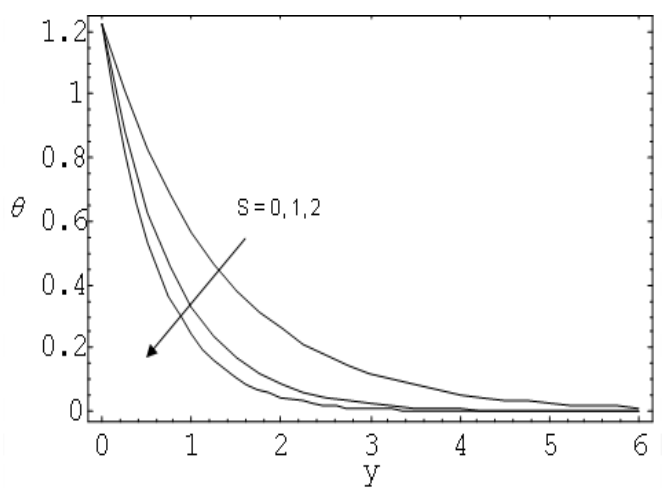

Fig. 2. Temperature profiles for different heat generation parameters with $n=0.1, t=1, \varepsilon=0.2$, $U_{p}=0.5, A=0.5, \operatorname{Pr}=0.7, G r=2.0, K=0.5$ and $M=2.0$

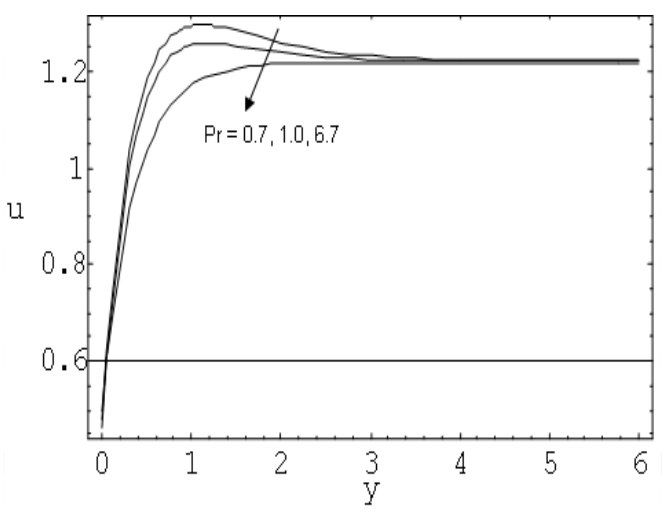

Fig. 3. Velocity profiles for different Prandtl numbers with $n=0.1, t=1, \varepsilon=0.2, U_{p}=0.5, A=0.5$,

$$
S=-1.0, G r=2.0, K=0.5 \text { and } M=2.0
$$

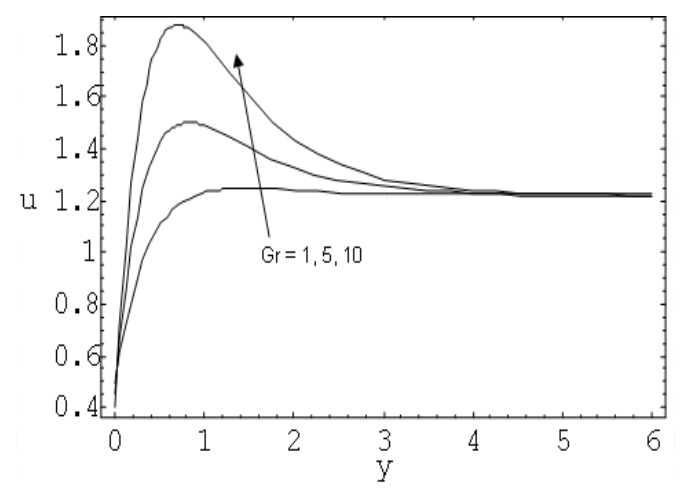

Fig. 4. Velocity profiles for different Grashof numbers $n=0.1, t=1, \varepsilon=0.2, U_{p}=0.5, A=0.5$,

$S=-1.0, \operatorname{Pr}=0.7, K=0.5$ and $M=2.0$

We have plotted the curves for velocity profile for different values of heat generation parameters $\mathrm{S}$ in Fig. 5. From this, we infer that when $S$ increases from zero, the velocity decreases. The velocity is minimum when $S=10$. Figure 6 plots the velocity profile against the 
spanwise co-ordinate y for different Hartmann numbers M.

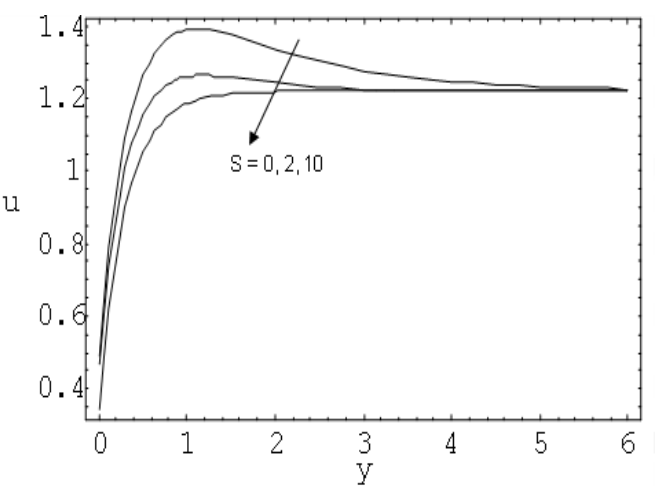

Fig. 5. Velocity profiles for different heat generation parameters $n=0.1, t=1, \varepsilon=0.2, U_{p}=0.5$,

$$
\begin{gathered}
A=0.5, \operatorname{Pr}=0.7, G r=2.0, K=0.5 \text { and } \\
M=2.0
\end{gathered}
$$

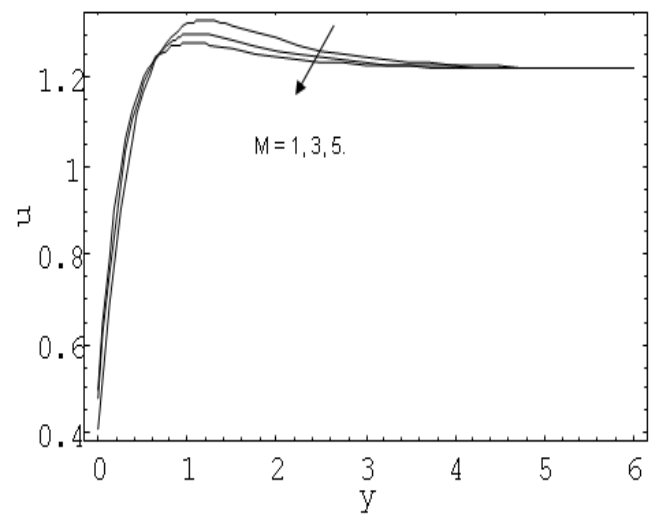

Fig. 6. Velocity profiles for different Hartmann numbers with $n=0.1, t=1, \varepsilon=0.2, U_{p}=0.5$, $A=0.5, \operatorname{Pr}=0.7, G r=2.0, K=0.5$ and $S=0.1$

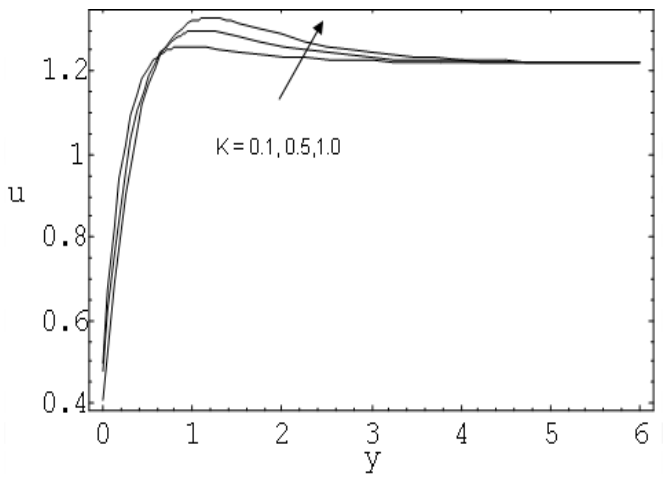

Fig. 7. Velocity profiles for different permeability of the porous medium $n=0.1, t=1, \varepsilon=0.2$, $U_{p}=0.5, A=0.5, \operatorname{Pr}=0.7, G r=2.0$ and

$$
M=2.0
$$

This illustrates that the velocity decreases as the existence of magnetic field becomes stronger. This conclusion agrees with the fact that the magnetic field exerts retarding force on the free- convection flow. From Fig. 7, we observe that the velocity increases with increase values of the permeability $\mathrm{K}$ of the porous medium. It is seen that the velocity attains the maximum value when $K=1.0$ for all the values of $K$, the velocity starts from zero initially and increases gradually, attains the corresponding peaks and then reduces until the free-stream velocity is attained.

Physically this result can be achieved when the holds of the porous medium are very large so that the resistance of the medium may be neglected.

\section{CONCLUSION}

The problem of MHD convective heat transfer past a semi-infinite moving plate with variable suction and heat generation has been studied. The governing equations are developed and transformed into a system of ordinary differential equations by perturbation technique and are solved analytically. The details of velocity and temperature fields are presented for various values of parameters of the problem. We observe that, when the magnetic parameter increases, the velocity decreases whereas when Grashof number and the heat generation parameter increases, the velocity increases. It is hoped that the present work will serve to understand more complex problems involving the various physical effects investigated in this presentation.

\section{REFERENCES}

Afify, A.A. and N. S.Elgazery (2012). Lie group analysis for the effect of chemical reaction on MHD stagnation-point of heat and mass transfer towards a heated porous stretching sheet with suction or injection, Nonlinear Analysis: Modelling and Control, 17, 1-15.

Amkadni,M., A. Azzouzi and Z. Hammouch (2006). On the exact solutions of laminar MHD flow over a stretching flat plate, Communications in Nonlinear Science and Numerical Simulation.

Chamkha, A.J. (1998). Unsteady Hydromagnetic Flow and Heat Transfer on a Non-Isothermal Stretching Sheet Immersed in a Porous Medium, International Communications in Heat and Mass Transfer, 25, 899-906,

Chamkha, A.J. and C. Issa (1999). Mixed Convection Effects on Unsteady Flow and Heat Transfer Over a Stretched Surface, International Communications in Heat and Mass Transfer, 26(5), 717-728.

Chamkha, A.J., H.S. Takhar and G. Nath (1999). Unsteady Flow and Heat Transfer on a Semiinfinite Flat Plate with an Aligned Magnetic Field. International Journal of Engineering Science, 37, 1723-1736.

Chamkha, A.J. (2004). Unsteady MHD convective heat and mass transfer past a semi-infinite vertical permeable moving plate with heat absorption, Int. J. Eng. Sci., 42, 217-230. 
Ferdows, M., M.Z.I. Bangalee, J.C. Crepeau and M.A. Seddeek (2011). The effect of variable viscosity in double diffusion problem of MHD from a porous boundary with Internal Heat Generation, Progress in Computational Fluid Dynamics, 11(1), 54 - 65.

Kabeir, S.M.M.EL., A.M. Rashad and R. Subba Gorla (2007). Unsteady MHD combined convection over a moving vertical sheet in a fluid saturated porous medium with uniform surface heat flux, Mathematical and Computer Modeling, 46, 384397.

Kim, Y.J. (2000). Unsteady MHD convective heat transfer past a semi-infinite vertical porous moving plate with variable suction, Int. J. Eng. Sci., 38, 833-845.

Muthucumaraswamy, R. and P. Ganesan (1998). Unsteady flow past an impulsively started vertical plate with heat and mass transfer, Journal of Heat and Masstransfer, 34, 187-193.

Ogulu,A. and J.Prakash (2006). Heat transfer to Unsteady MHD flow past an infinite moving vertical plate with variable suction, Phys. Scr. 74 232-239.

Ouaf, M.E.M. (2005). Exact solution of thermal radiation on MHD flow over a stretching porous sheet, Appl. Math. Comput., 170,1117-1125.
Raptis, A.A. (1986). Flow through a porous medium in the presence of the magnetic field, Int. J. Energy Res., 10, 97-100.

Seddeek, M.A. and F.A. Salama (2007). The effects of temperature dependent viscosity and thermal conductivity on unsteady MHD convective heat transfer past a semi-infinite vertical porous moving plate with variable suction, Comput. Mat. Sci., 40, 186-192.

Sivasankaran, S., M. Bhuvaneswari and M. Ferdows (2009). Lie group analysis of natural convection heat and mass transfer in an inclined surface with chemical reaction, Nonlinear Anal., Hybrid Syst., $3,536-542$.

Suneetha, S., N. Bhaskar Reddy and V. Ramachandra Prasad (2011).Radiation and Mass Transfer Effects on MHD Free Convective Dissipative Fluid in the Presence of Heat Source/Sink, International Journal of Applied Fluid Mechanics, 4(1), 107113,

Toki, C.J. and J.N. Tokis (2007). Exact solutions for the unsteady free convection flows on a porous plate with time - dependent heating, ZAMM., 87(1), 413 